\title{
Investigation of formamidinium and guanidinium lead tri-iodide powders as precursors for solar cells
}

\author{
L. Dimesso*, ${ }^{1}$ A. Quintilla, ${ }^{2}$ Y.-M. Kim, ${ }^{1}$ U. Lemmer ${ }^{2}$ and W. Jaegermann ${ }^{1}$ \\ ${ }^{1}$ Technische Universitaet Darmstadt, Materials Science Department, Jovanka-Bontschits-Strasse 2, D-64287 \\ Darmstadt,Germany \\ ${ }^{2}$ Karlsruhe Institute of Technology (KIT), Institute of Microstructure Technology, Hermann-von-Helmoltz 1, D- \\ 76344 Karlsruhe (Germany)
}

\begin{abstract}
Formamidinium and guanidinium lead tri-iodide powders $\left(\mathrm{FAPbI}_{3}\right.$ and $\mathrm{GUAPbI}_{3}$ respectively) were prepared by self-organization processes in solutions. The XRD analysis detected the formation, at room temperature, of a trigonal structure $\left(\alpha-\mathrm{FAPbI}_{3}\right.$, space group P3m1), of a (non-perovskitic) hexagonal structure $\left(\delta-\mathrm{FAPbI}_{3}\right.$, space group P63mc) for $\mathrm{FAPbI}_{3}$, and the presence of orthorhombic (space group Pnma) and hexagonal structures for $\mathrm{GUAPbI}_{3}$ respectively. The morphological investigation revealed the growth of aggregated $\left(\alpha-\mathrm{FAPbI}_{3}\right.$ phase $)$ and stripe-like crystallites $\left(\mathrm{GUAPbI}_{3}\right)$ respectively. The infrared spectra (IR) confirmed the strong influence of additional $\mathrm{C}-\mathrm{N}$ bonds on vibrational properties of GUA-containing system. Optical absorption measurements indicated that $\alpha-\mathrm{FAPbI}_{3}$ and $\mathrm{GUAPbI}_{3}$ systems reveal direct energy band gaps of $1.42 \mathrm{eV}$ and $1.92 \mathrm{eV}$ respectively at room temperature. Photoluminescence measurements revealed emissions in the infra-red (IR) (for $\alpha-\mathrm{FAPbI}_{3}$ ) and visible (Vis) ranges (for $\mathrm{GUAPbI}_{3}$ ). Films formation of $\mathrm{GUAPbI}_{3}$ was demonstrated, and solar cells devices were fabricated with average power conversion of $0.3 \%$.
\end{abstract}

Keywords: Formamidinium lead iodide, guanidinium lead iodide, solar cell, powder, infrared

*Corresponding Author

Dr. L. Dimesso

Technische Universitaet Darmstadt, Materials Science Department

Jovanka-Bontschits-Strasse 2

D-64287 Darmstadt (Germany)

Fax: +49 6151 166308; Tel.: +496151 1669667

E-mail:1dimesso@surface.tu-darmstadt.de 


\section{Introduction}

The very significant manufacturing costs reduction of photovoltaic modules assures a future for the PV use within the renewable energy sources and recent forecasts anticipate already that PV will contribute by a third to the electricity generation in the near future [1,2]. The new generation of mixed organic-inorganic halide perovskites has shown to be an outstanding candidate for boosting the module efficiencies as a tandem partner with high efficiency established materials (as silicon or thin-film $\mathrm{Cu}(\mathrm{In}, \mathrm{Ga}) \mathrm{Se}_{2}$ based materials) and for lowering the processing costs due to their ease of fabrication [3]. Further optical and electrical key properties of these hybrid perovskites besides the easy processing thereof are the high absorption coefficient, the band gap tunability, the low non-radiative carrier recombination rates and the high luminescence yield: the applications and use of perovskites range from solar cells and photodetectors to light emitting devices as organic based LEDs or lasing devices. Perovskites are materials described by the formula $\mathrm{ABX}_{3}$, where $\mathrm{X}$ is an anion, $\mathrm{A}$ and $\mathrm{B}$ are cations of different sizes (A being larger than $\mathrm{B}$ ). Among these hybrids, self-organized 3D networks of $\mathrm{R}^{-\mathrm{MX}_{3}}$ (R-organic amine and MX-metal halide) type perovskites have attracted much attention because of their unique crystal structures and the modified optical properties [4-10]. The perovskite structure is generally described as three-dimensional arrangement of a cornersharing octahedral $\mathrm{BX}_{6}$ unit, with the $\mathrm{A}$ ion placed in the cubo-octahedral interstices: in the ideal case, this structure is cubic.

Although hybrid perovskites have been widely investigated for their photoactive properties, the recent reports of $\mathrm{CH}_{3} \mathrm{NH}_{3} \mathrm{PbI}_{3}$ containing solar cell devices have focused both on fabrication procedures (single step, sequential deposition and vapor deposition) and different device architectures (planar vs mesostructured). This has led to some scrutiny about the role played by the $\mathrm{CH}_{3} \mathrm{NH}_{3} \mathrm{PbI}_{3}$ perovskite layer, which has been shown to exhibit both electron and holes transporting behavior. The high efficiencies of devices containing $\mathrm{CH}_{3} \mathrm{NH}_{3} \mathrm{PbI}_{3}$ can be traced to its high absorption coefficient, good electrical transport properties, and favorable band gap $(1.55 \mathrm{eV})$, which is close to the optimum value for a single junction solar cell [11]. However, the chemical modification of the X site anions (e.g., substitution of I for Br) show an increase of the band gap while modulating the power conversion efficiencies (PCE). Similarly, the substitution of the organic cation $\left(\mathrm{CH}_{3} \mathrm{NH}_{3}{ }^{+}\right)$with a longer chain alkyl group (for example $\mathrm{C}_{2} \mathrm{H}_{5} \mathrm{NH}_{3}{ }^{+}$) resulted in a larger band gap and lower power conversion efficiency (PCE) (2.4\%) [11]. In this work we

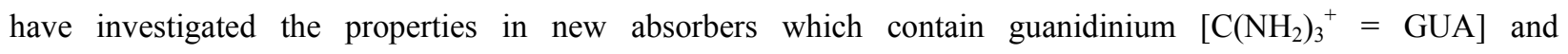


formamidinium cation $\left[\mathrm{HC}\left(\mathrm{NH}_{2}\right)_{2}{ }^{+}=\mathrm{FA}\right]$. Then we compared their properties with those of containing methylammonium cation $\left(\mathrm{MA}=\mathrm{CH}_{3} \mathrm{NH}_{3}{ }^{+}\right)$in the perovskite structure.

The FA-cation containing systems are intensively investigated due to the low electronic band gap $\left(\mathrm{E}_{\mathrm{g}}=1.47 \mathrm{eV}\right.$ on spin-coated layers) which is closer to the optimum value of $\sim 1.4 \mathrm{eV}$ and presents $\mathrm{FAPbI}_{3}$ as an appealing candidate for photovoltaic applications, as it displays an extended absorption of light compared to the $\mathrm{MAPbI}_{3}$ analogue. Formamidinium ion has a slightly larger ionic radius than the methylammonium group $\left(R_{\mathrm{A}}=0.18 \mathrm{~nm}\right.$ for $\mathrm{MA}^{+}$and $0.19-0.22 \mathrm{~nm}$ for $\mathrm{FA}^{+}$respectively. ${ }^{2}$ The larger ionic radius is expected to lead to in an increase in perovskite tolerance factor $(t)$. An increase in $t$ while maintaining the $\mathrm{ABX}_{3}$ perovskite structure generally leads to an increase in symmetry, with an expected reduction in electronic band gap [12]. On the other hand, aqueous guanidinium salts are widely employed by biophysicists and researchers of biomolecules and colloids in general in order to study specific ion effects. The ionic radius of the GUA-cation $\left(\mathrm{R}_{\mathrm{a}}=0.21 \pm 0.02 \mathrm{~nm}\right)[13]$ lies in the same range of the $\mathrm{FA}^{+}$-cation, consequently very similar properties are expected. Indeed until now, no scientific references have been found on the photoluminescence properties of guadinidium containing lead iodide. The main purpose of this work is the investigation of the structural, morphological, optical and electronic properties of $\mathrm{GUAPbI}_{3}$ and $\mathrm{FAPbI}_{3}$ which can be used as precursors for dispersions to thin films devices and compared to those of $\mathrm{MAPbI}_{3}$ systems.

\section{Experimental}

Formamidinium and guanidinium lead iodide powders were prepared by self-organization processes under very similar conditions reported for the preparation of methylammonium containing systems [14]. $\mathrm{Pb}\left(\mathrm{CH}_{3} \mathrm{COO}\right)_{2} * 2 \mathrm{H}_{2} \mathrm{O}$ (lead acetate dehydrate, Alfa Aesar), HI (47\% wt., iodic acid stabilized with 1.5\% wt hypophosphorous acid, Alfa Aesar), $\mathrm{NH}_{2} \mathrm{C}(=\mathrm{NH}) \mathrm{NH}_{2} \cdot 0.5\left(\mathrm{H}_{2} \mathrm{CO}_{3}\right)$ guanidine carbonate (Sigma-Aldrich) and formamidinium iodide $\left(\mathrm{HC}\left(\mathrm{NH}_{2}\right)_{2} \mathrm{I}\right.$, Dyesol Australia Pty Ltd) were used as precursors for the preparation of $\mathrm{GUAPbI}_{3}$ and $\mathrm{FAPbI}_{3}$ without further purification. Stripe-like crystallites (up to $10-12 \mathrm{~mm}$ for $\mathrm{GUAPbI}_{3}$ ) and needle-like crystallite (up to $8 \mathrm{~mm}$ for $\mathrm{FAPbI}_{3}$ ) were grown by cooling aqueous solutions from $115-120^{\circ} \mathrm{C}$ to room temperature. The structural analysis of the samples was performed, after thoroughly grinding, by X-ray powder diffraction (XRD) using a D8 Bruker powder diffractometer $(\mathrm{Cu} \mathrm{K \alpha 1}+\mathrm{Cu} \mathrm{K \alpha 2}$ radiation) with a theta / 2 theta Bragg-Bentano configuration. The diffractometer is equipped with an Energy Dispersion Detector $\mathrm{Si}(\mathrm{Li})$ to minimize the fluorescence effects. A 
scanning electron microscope (SEM) Philips XL 30 FEG was used to investigate the morphology of the samples. Optical diffuse-reflectance measurements were performed using a Perkin Elmer UV/VIS/NIR doublemonochromator spectrometer operating from $200 \mathrm{~nm}$ to $2500 \mathrm{~nm}$. $\mathrm{BaSO}_{4}$ was used as a non-absorbing reflectance reference. The generated reflectance-versus-wavelength data were used to estimate the band gap of the material by converting reflectance to absorbance data according to the Kubelka-Munk equation: $\alpha / S=(1-R)^{2} / 2 R$, where $R$ is the reflectance and $\alpha$ and $S$ are the absorption and scattering coefficients, respectively $[15,16]$. The infrared (IR) absorption was measured with a Varian Fourier-Transformed-Infrared (FTIR) spectrometer Model IR1001M010. The spectral slit width was $1 \mathrm{~cm}^{-1}$ and the investigated spectral range was between 6000 and $400 \mathrm{~cm}^{-1}$. The photoluminescence (PL) measurements were performed by a VARIAN Cary Ellipse fluorescence spectrophotometer equipped with a Xe-arc as emission source. The emission was measured in the 400-900 nm range. The Guacontaining compound was also measured by an additional set-up: the sample was excited at $355 \mathrm{~nm}$ with a diodepumped, actively Q-switched, frequency-tripled $\mathrm{Nd}_{\mathrm{YVO}} \mathrm{YV}_{4}$ laser (AOT-YVO-20QSP) with $9 \mu \mathrm{Jcm}^{-2}$. Photoluminescence was fiber-coupled into a spectrograph (Acton Research SpectraPro 300i) and detected with an intensified CCD detection array (PiMAX512, Princeton). All measurements were performed at room temperature.

\section{Results and discussion}

Crystallographic stability and probable structure of perovskites can be deduced by considering the "Goldschmidt's tolerance factor" $(t)$ [17] and the "Li's octahedral factor" $(\mu)$ [18]. The tolerance factor was introduced to evaluate ionic size mismatches which the perovskite-structure will tolerate until a different structuretype is formed (see equation (1) in Support Information) in the perovskite $\mathrm{ABX}_{3}$. For values of $t$ in the range 0.91.0 , mostly cubic perovskites are found, whereas values of $0.80-0.89$ predominantly lead to distorted perovskites which can be further classified by using Glazer's concept of octahedral tilting [19]. Below $\sim 0.80$, other structures such as the ilmenite-type $\left(\mathrm{FeTiO}_{3}\right)$ are more stable due to the similar sizes of the cations $\mathrm{A}$ and $\mathrm{B}$. Values of $t$ larger than 1.0 lead to hexagonal structures where layers of face-sharing octahedra are introduced into the structure. Moreover, in the case of hybrid perovskites we are dealing with molecular cations, where varying bond lengths due to hydrogen-bonding interactions make it difficult to define an ionic radius. Recently, Kieslich [20] extended the tolerance factor concept to organic perovskites. The authors used crystallographic data from known perovskite-like hybrid frameworks to estimate a consistent set of "effective ionic radii" $\left(r_{\text {Aeff }}\right)$ for different organic ions given by the contribution of $r_{\text {mass }}$, being the distance between the centre of mass of the molecule and the atom with the largest 


\begin{tabular}{ccccccc}
\hline $\mathbf{A}$ & $\mathbf{r}_{\text {Aeff }}(\mathbf{p m})$ & $\mathbf{t}$ & $\boldsymbol{\mu}$ & $\boldsymbol{\lambda}_{\text {on-set }}(\mathbf{n m})$ & $\mathbf{E}_{\mathbf{g}}(\mathbf{e V})^{\mathbf{a}}$ & $\mathbf{E}_{\mathbf{g}}(\mathbf{e V})^{\mathbf{b}}$ \\
\hline MA & 217 & 0.912 & 0.541 & 816 & 1.52 & 1.53 \\
FA & 253 & 0.987 & 0.541 & 880 & 1.41 & 1.42 \\
GUA & 278 & 1.039 & 0.541 & 645 & 1.92 & 2.16
\end{tabular}

Table 1. Summary of the values of $t$ and $\mu$ factors, optical absorbance edges, band gap energies. The values of $r_{\text {Aeff }}$ and $t$ are from Ref. [20] and confirmed by our own calculations. The band gap energy values are derived from: a) Figure 4a; b) Figure 4b according to a model described in [14] for the investigated organic-inorganic lead iodide perovskites. The values in brackets for guanidinium containing systems are derived from Figure 5B. The data on the methylammonium (MA) containing compounds are from Ref. [14].

distance to the centre of mass, excluding hydrogen atoms, and $r_{\text {ion }}$ being the corresponding ionic. The $r_{\text {Aeff }} t$ and $\mu$ factors of $\mathrm{FAPbI}_{3}$ and $\mathrm{GuaPbI}_{3}$ systems are summarized in Table 1. According to the values reported in Table 1, $\mathrm{FA}^{+}$- and $\mathrm{GUA}^{+}$-containing systems would satisfy the criteria for the formability of halide perovskites. Indeed the formation of perovskite structures in MA and FA containing systems has been widely reported in the literature, but we have not found any information on the GUA-containing system. The tolerance and octahedral factors are necessary but not sufficient conditions for $\mathrm{ABX}_{3}$ halide perovskite formability, the structural and morphological characterizations are the best tools to confirm the goodness of the predictions only.

The X-ray diffractogramms of FA- and GUA-containing perovskites are shown in Figure S1a and Figure 1 respectively. Indeed, replacement of $\mathrm{MA}^{+}$with slightly larger $\mathrm{FA}^{+}$ions and much lower dipolar moment [21] stabilizes a perovskite structure with either trigonal structure (black color) (called $\alpha-\mathrm{FAPbI}_{3}$, space group $P 3 m 1$ ) [22] as shown in Figure S1A or non-perovskite structure with hexagonal structure (yellow color) $\left(\delta-\mathrm{FAPbI}_{3}, \mathrm{space}\right.$ group $\mathrm{P}_{3} m c$ ) shown in Figure S2A. According to our observations during the preparation experiments, the black phase is stable at high temperatures $\left(>90^{\circ} \mathrm{C}\right)$ while at lower temperatures the black phase fully converts into the yellow phase inside the mother liquor. When the products are separated from the solvent and dried at room temperature, they interconvert after annealing at $\mathrm{T}=200^{\circ} \mathrm{C}$ for $\mathrm{t}=8 \mathrm{hr}$ under flowing nitrogen atmosphere, the $\alpha$ $\mathrm{FAPbI}_{3}$ phase becomes more stable. Recently, Weller et al. [23] reported that the structure of black FAPI, at room 
temperature, consists of a cubic lead iodide, $\left[\mathrm{PbI}_{3}\right]^{-}$perovskitic framework surrounding orientationally disordered, planar $\mathrm{FA}^{+}$-cations, Indeed, the authors developed a structure model for cubic FAPI, from neutron diffraction and XRD analysis, using non-tilted $\mathrm{PbI}_{6}$ octahedra in the space group Pm-3m with the FA cations formally disordered over 24 orientations with the perovskitic $\left[\mathrm{PbI}_{3}\right]^{-}$framework. In this structure the $\mathrm{C}-\mathrm{H}$ bonds point directly toward the cube face (thereby minimizing $\mathrm{CH} \cdots \mathrm{I}$ interactions), and the $\mathrm{C}-\mathrm{N}$ bonds directed toward adjacent cube faces. This allows the $-\mathrm{NH}_{2}$ groups to be orientated toward the unit cell edges with the potential formation of $\mathrm{NH} \cdots \mathrm{I}$ hydrogen bonds. Unlike $\mathrm{MA}^{+}$-ions and $\mathrm{FA}^{+}$-ions containing compounds, there is very few information in the literature on $\mathrm{GUAPbI}_{3}$ materials. A typical XRD diffractogram of yellow crystallites obtained during the preparation of $\mathrm{GUAPbI}_{3}$ materials are shown in Figure 1. In the solid state, the guanidinium cation may be planar or slightly distorted with symmetry approximately $D_{3 h}$ [24]. Besides, it is a donor of six hydrogen atoms and, thus, can be involved in hydrogen bonds. The high symmetry and the ability to form hydrogen bonds are important factors determining the structure and the properties of the crystals. Szafranski $[25,26]$ reported that the tri-iodide undergoes two first-order phase transitions at 255 and $432 \mathrm{~K}$ respectively during his investigation of phase instabilities in

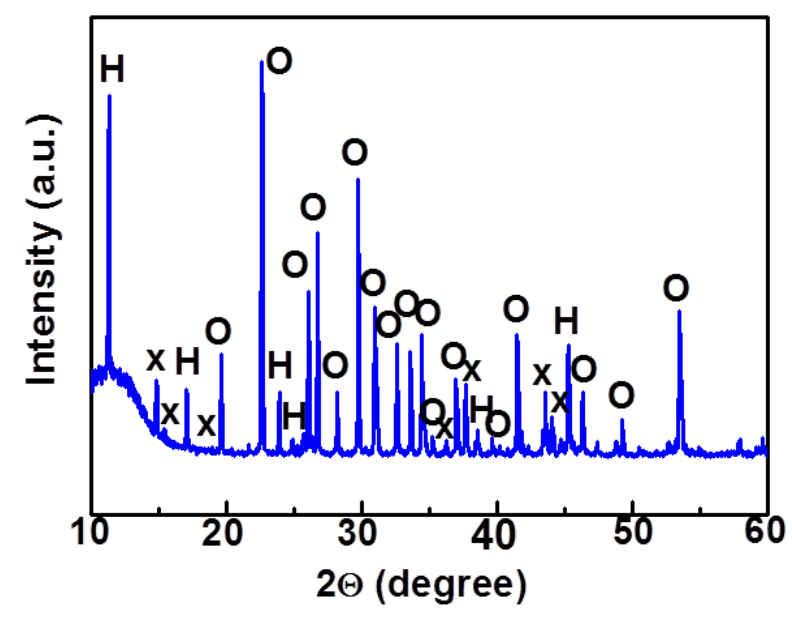

Figure 1. XRD-pattern of $\mathrm{GUAPbI}_{3}$ system. The labels indicate $\mathrm{O}$

$=$ orthorhombic $\mathrm{GUAPbI}_{3}, \mathrm{H}=$ hexagonal $\mathrm{GUAPbI}_{3}$ and $\mathrm{x}=$ guanidinium iodide respectively.

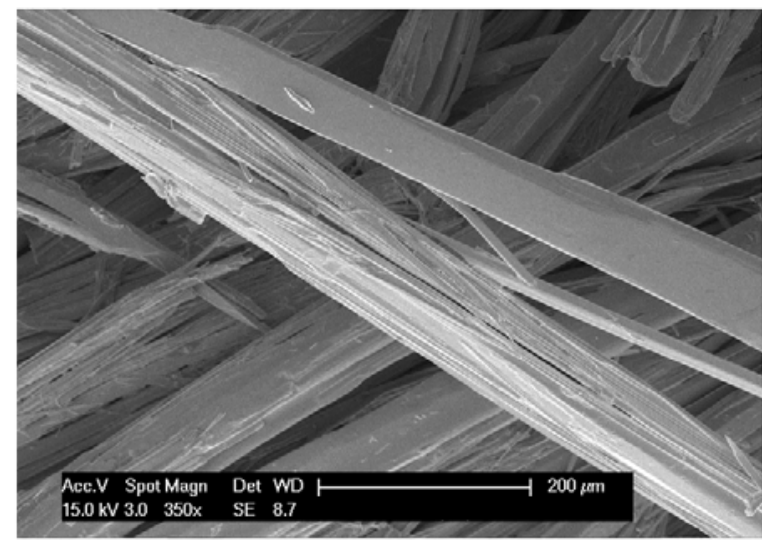

Figure 2. Typical SEM image of $\mathrm{GUAPbI}_{3}$ system.

guanidinium and halogenoplumbates (II) by differential thermal analysis (DTA). The transition from the phase II to the high temperature phase I was observed near $432 \mathrm{~K}$. The crystal structure of phase II has been determined by Xrays [27]. In this phase the crystal has orthorhombic symmetry of $\mathrm{KCdCl}_{3}$ type, space group Pnma. The $\mathrm{Pb}^{2+}$ ions 
are octahedrally coordinated and the $\mathrm{Pbl}_{6}$ octahedra are arranged in double chains with chain axis parallel to the $b$ axis. The inorganic chains are separated by guanidinium cations. The large transition entropy proved that this phase transition is of the order-disorder type. In our case, the formation of the orthorhombic phase (indicated with "O" in Figure 1) as main phase was detected. Furthermore, crystalline reflections of the $\mathrm{GUAPbI}_{3}$ hexagonal phase (indicated with " $\mathrm{H}$ " in Figure 1) and of guanidinium iodide (indicated with "x" in Figure 1) were observed. We also observed that the phase I exhibits metastable properties. At ambient pressure and temperature, the crystals transform from this phase to phase II after several hours. An interesting feature is a change in the color of the crystals; when they convert to the phase I, they become orange-reddish as reported also by Szafranski [25]. This can be explained by displacement of the iodine atoms as well as by the by modifications of the electronic structure of the $\mathrm{PbI}_{6}$ octahedra. A gradual restoration of the yellow color, characteristic for phase II, was observed when the samples previously heated to the phase I temperature, were cooled and left at room temperature. To obtain pure materials, solution processes are very suitable. Our synthetic procedure (self-organization process) is a modification of a reported method in aqueous solutions [14] and gives no observable impurities while it allows for easier manipulation of the materials. The results of the morphological investigation confirmed the influence of the structural properties on crystallite habits of the prepared materials as shown in Figure 2 and Figure S1B respectively. The crystallites of the $\mathrm{FAPbI}_{3}$ black phase (Figure $\mathrm{S1B}$ ) revealed the formation of aggregates due to the quenching (very fast cool down to room temperature) and fast separation from the mother liqueur. Indeed, the habit of the material can even vary from aggregate crystallites when the crystallization occurs at high temperature (ca. $120^{\circ} \mathrm{C}$ in our case) under vigorous stirring, to discrete polyhedral crystals when crystallization occurs by cooling slowly to room temperature and without stirring. This fact is confirmed by the formation and growth of stripe-like crystallites of the GUAcontaining lead iodide up to 10-12 $\mathrm{mm}$ as shown in Figure 2.

Infrared spectra (IR) of $\mathrm{FAPbI}_{3}$ and $\mathrm{GUAPbI}_{3}$ systems are shown in Figure 3a-b respectively, and a summary of the vibrational group frequencies is shown in Table S1. The FT-IR absorption spectra, recorded in the wavenumber range between $v / 6000 \mathrm{~cm}^{-1}$ and $v / 400 \mathrm{~cm}^{-1}$, give information on the vibrational behavior of the "organic" groups in the systems only. Information on the lattice dynamical properties of the Pb-I groups in the octahedral coordination can be obtained by measuring at higher frequencies (lower than $v / 400 \mathrm{~cm}^{-1}$ ) [28]. By observing the FT-IR spectra of the prepared compounds, some points are to note. First, the spectra are influenced by the $\mathrm{C}-\mathrm{N}$ bonds in the organic cations as well as by the stronger electron delocalization in FA and GUA cations as proved by the disappearance of 


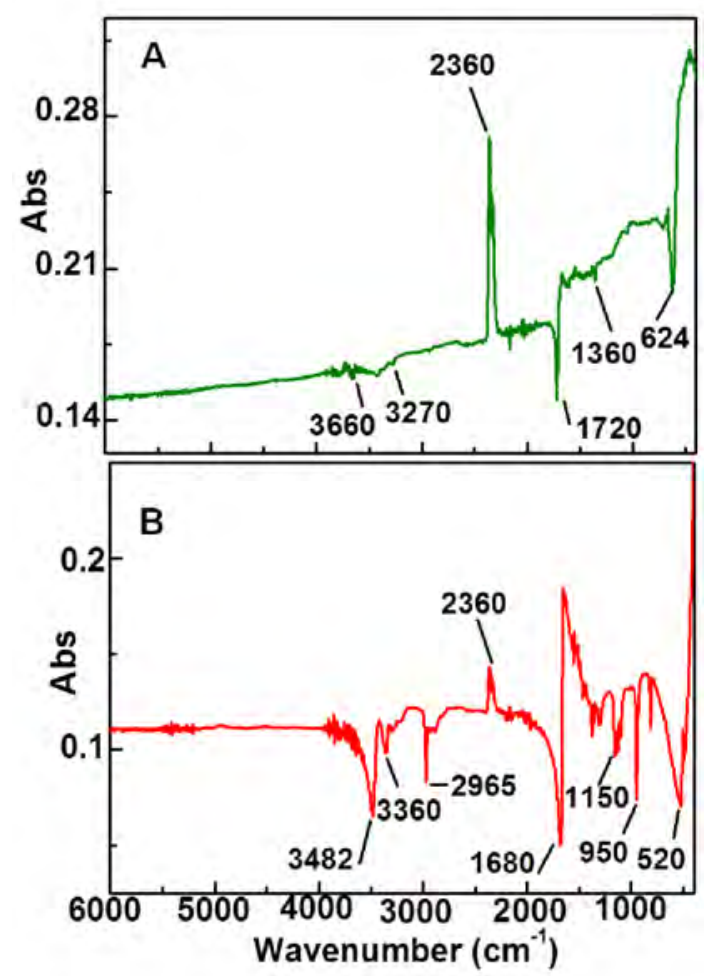

Figure 3. FT-IR spectra, measured at room temperature, for a) $\mathrm{FAPbI}_{3}$ (black phase) and b) $\mathrm{GUAPbI}_{3}$ system respectively. The relevant vibrational features have been labeled.


Figure 4. A) Normalized absorption spectra and B) plots $(\alpha h v)^{2}$ for direct transitions in $\mathrm{FAPbI}_{3}$ system (in inset $\mathrm{GUAPbI}_{3}$ ), where $\alpha$ is absorption coefficient and $h v$ is photon energy. Band gaps $E_{g}$ are obtained by extrapolation to $\alpha=0$. The data are reported in Table 1 . The curves of $\mathrm{MAPbI}_{3}$ (black dots, Ref. [14]) have been added for comparison.

the "fang vibrations" $\left(3761-3468 \mathrm{~cm}^{-1}\right)$ typical of primary ammines from the formamidinium IR-spectrum (Figure 3a). Fundamental vibrations above $v / 4000 \mathrm{~cm}^{-1}$ have not been observed in FT-IR spectra. Second, a more complex delocalization of the lone pair electrons of the nitrogen atoms with the vacant $\mathrm{p}$ orbital of the carbon atom IRspectrum has been observed in the guanidinium containing lead iodide system (Figure 3b). The guanidinium ion is derived by protonation from the very strong base guanidine, $\mathrm{NH}-\mathrm{C}\left(\mathrm{NH}_{2}\right)_{2}\left(\mathrm{pK}_{\mathrm{b}}=\mathbf{1 3 . 6}\right.$ at $\left.25^{\circ} \mathrm{C}\right)$. In terms of a molecular orbital description, the planarity of the $\mathbf{C N}_{3}$ skeleton corresponds to $s p^{2}$ hybridization of the carbon atom. The $\mathrm{CN}$ bonds acquire partial double-bond character on this picture because of some degree of interaction considered as $\mathrm{C}^{+}$. Detailed experimental and theoretical studies on the assignments of the IR vibration frequencies of the guanidinium ion have been reported in literature $[24,29,30]$ during the investigation of different guanidinium salts. The authors compared their data with those obtained from Raman and XRD measurements previously published. A difference in the values of vibration frequencies can be explained by the different interactions 
guanidinium - iodine and guanidinidium - lead - iodine systems. On the other hand, vibrational properties of formamidine and FA+ cations are not well understood and a reliable assignment of vibrational bands for these groups is still needed. Recently Kucharska et al. [31] reported the results of DFT calculations for a formamide molecule and formamidinium cation and compared them with experimental values obtained from synthesized metal formates. FA+ cation has 18 vibrational degrees of freedom due to the 6 vibrations of the $\mathrm{NH}_{2}$ group. Furthermore, FA+ cation has two equal $\mathrm{C}-\mathrm{N}$ bonds and, therefore, stretching modes of these bonds should be denoted as symmetric stretching and antisymmetric stretching modes (at $3800 \mathrm{~cm}^{-1}$ and $3400 \mathrm{~cm}^{-1}$ respectively). The comparison of our experimental results shows that the largest difference between harmonic and anharmonic wavenumbers occurs for the stretching modes of the $\mathrm{NH}_{2}$ and $\mathrm{CH}$ groups (see Table $\mathrm{S} 1$ ). This result indicates strong anharmonic nature of these vibrational modes. Moreover the very weak signal of " $\mathrm{NH}_{2}$-twisting modes" would indicate that the FA-cations are not free to move due to the formation of H-bridge and consequent interaction with iodine ions of the $\mathrm{PbI}_{6}$ octhedra.

The differences in the structural and morphological features of the investigated organic-inorganic perovskites, have a dramatic influence on the optical properties of the prepared materials. In Figure 4A, the optical properties of $\mathrm{FAPbI}_{3}$ and $\mathrm{GUAPbI}_{3}$ systems (additionally those of $\mathrm{MAPbI}_{3}[14]$ for comparison) are shown. The MA- and FAcontaining systems display a strong, abrupt absorption in the near-infrared (N-IR) spectral region whereas the GUAcontaining system display an absorption in the visible (VIS) region. Assuming a bulk-like behavior of the prepared samples, the values of the band energy gap $\left(E_{g}\right)$ have been calculated by using the relationship $E_{g} \bullet \lambda_{\text {on-set }}=$ const, in which $E_{g}$ is in $\mathrm{eV}$, const $=1239.84(\mathrm{eV} \bullet \mathrm{nm})$ and $\lambda_{\text {on-set }}(\mathrm{nm})$ is the frequency value at which the photons absorption begins, obtained by fitting the linear part of the absorbance spectrum [32]. The data at wavelengths lower than $805 \mathrm{~nm}$ for $\mathrm{FAPbI}_{3}$ have been omitted due to dispersion and diffusion effects. The values of the energy gaps are reported in Table 1. By observing Table 1 some important points are to note. First, as shown in Figure 4A, a dependence of the band-gap energy upon the structural symmetry of the perovskite can be observed. In addition, the pre-gap region does not show strong absorption tails indicating that high quality materials with low defect concentration have been prepared. The band gap values of $\mathrm{FAPbI}_{3}\left(1.41 \mathrm{eV}\right.$ ) (and $\mathrm{MAPbI}_{3}, 1.52 \mathrm{eV}$ ) reported in Table 1 are lower than those reported in literature, whereas we have not found out any further data concerning the band energy gap values of $\mathrm{GUAPbI}_{3}$ system. In our case, $\mathrm{GUAPbI}_{3}$ perovskite showed a shifted absorption onset 
$(645 \mathrm{~nm}$, corresponding to $1.92 \mathrm{eV})$. As nano-particles in powders tend to agglomerate and consequently to develop bulk-like bands, the relationship between the adsorption coefficient $(\alpha)$ near the absorption edge and the optical band gap $\left(E_{g}\right)$ for direct interband transitions obeys a relationship as previously reported [14].The plots derived from the relationship, leads to graphics as shown in Figure 4B. By extrapolating to $\alpha=0$, the plots revealed a behavior of a direct semiconductor of $\mathrm{FAPbI}_{3}$ and $\mathrm{GuaPbI}_{3}$ with band energy gaps of $1.42 \mathrm{eV}$ and $2.16 \mathrm{eV}$ respectively. Second, replacement of methylammonium by formamidinium, effectively lead to a red-shifted absorption onset. Solar cells fabricated with $\mathrm{FAPbI}_{3}$ showed an extended photocurrent generation onset on the red-wing of solar spectrum also and mixed MA-FA perovskites, showed tunable band gap by varying the relative MA/FA amount [33]. These results cannot be interpreted in terms of the different cation size, whereby MA $<$ FA $<$ GUA, should lead to a band gap reduction which is not the case. Giorgi et al. [21] theoretically examined the effects of the incorporation of GA cations into the lead iodide perovskite on the thermodynamic stability and electronic properties by means of density functional theory (DFT) calculations suggested that the increased value of the band-gap for $\mathrm{GUAPbI}_{3}$ is consistent with the fact that a more bulky cation $(\mathrm{GA}>\mathrm{FA})$ reduces the anti-bonding interactions between $\mathrm{Pb}$ and $\mathrm{I}$ orbitals forming the valence band maximum (VBM) and the conduction band minimum (CBM) of similar systems. In other words, the VBM (and to a lesser extent the CBM, where the presence of I orbitals is negligible) will be stabilized, with a subsequent band-gap opening. Furthermore, $\mathrm{MAPbI}_{3}$ crystallizes in the tetragonal $\mathrm{I} 4 / \mathrm{mcm}$ (or $I 4 / \mathrm{cm}$ ) space group at room temperature, characterized by a sizable tilting of the $\mathrm{PbI}_{6}$ octahedra, $\mathrm{FAPbI}_{3}$ exhibits a trigonal $P 3 m 1$ space group, which is close to a cubic structure whereas Grottel [27] has reported the preferential formation of orthorhombic structures for $\mathrm{GAPbI}_{3}$. Thus, one may also wonder whether the different crystal structures (tetragonal versus trigonal/pseudocubic) could play a role, beyond the different volume and/or cation size, in determining the observed variation of optical properties. Finally, the interaction between the organic cations and the inorganic matrix mainly takes place, beyond electrostatics, through hydrogen bonding between the acidic MA or FA hydrogen atoms and the perovskite iodides, which could also contribute to determine the materials properties. Guanidinium cations are planar-trigonal of high symmetry, $\mathrm{D}_{3 \mathrm{~h}}$, in crystals as well as in aqueous solutions. Mason [34], by reporting a neutron-diffraction with-isotopic-substitution (NDIS) $\left({ }^{15} \mathrm{~N}\right.$ and $\left.{ }^{14} \mathrm{~N}\right)$ study of a moderately concentrated aqueous solution of guanidinium chloride $\left(3.33 \mathrm{~mol}\right.$ in $\left.55.55 \mathrm{~mol}_{2} \mathrm{O}\right)$, showed that little hydration of the cation, in the sense of stable hydrogen bond formation between the cation and water molecules, takes place $(\mathrm{NH} \cdots \mathrm{O})$. The GUA-cation remains flat in the solution and the tetrahedral water structure around it seems to be little affected by its presence. 
The PL properties of $\mathrm{FAPbI}_{3}$ and $\mathrm{GUAPbI}_{3}$ systems, at room temperature, were measured using an excitation wavelength of $380 \mathrm{~nm}$. PL emissions were observed for all specimens (shown in Figure 5A). The emission wavelength of $\mathrm{FAPbI}_{3}$ (peak maximum at $\sim 803 \mathrm{~nm}$ ) is consistent with our optical absorption and with previous results reported in literature [22]. On the other hand, the PL-profile of $\mathrm{GUAPbI}_{3}$ was too weak for a correct interpretation of the PL-properties (Figure 5A-inset). In order avoid any confusing data with consequent misleading conclusions, PL properties of the guanidinium containing system were also determined by Nd-laser source with a monochromatic wavelength $(355 \mathrm{~nm}$ ). The results (shown in Figure 5b) revealed an emission at 667nm (which corresponds to $E_{g}=1.85 \mathrm{eV}$, very close to the theoretical predictions of $1.78 \mathrm{eV}$ [21]) and a PL-signal with two maxima was observed. An explanation for the presence of two peaks in these spectra is still to be cleared and whether it is due to different phases with different stoichiometries or to one phase with different crystalline structures and/or sublattices is still to be determined.

The next step of our investigation was the preparation of layers using the prepared powders as precursors. As example, ca. $0.20 \mathrm{~g}$ of $\mathrm{GUAPbI}_{3}$ have been dissolved in $1.80 \mathrm{~g}$ of DMSO, resulted in very homogeneous and stable dispersion with $10 \%$ wt of $\mathrm{GUAPbI}_{3}$ ). Typically, ca. $100 \mathrm{mg}$ of the dispersion have been dropped on fluorine- doped tin oxide coated (FTO-coated) glass substrates, then deposited by coating and finally thermally treated $\left(150^{\circ} \mathrm{C}\right.$ for 5 minutes) in order to evaporate the solvent. After the evaporation of the solvent, the formation of an orange-like layer was observed. Then, by decreasing the temperature in air the orange layer changed color and turned into yellow as happened by heating up and cooling down the powders. We concluded that this process favored the recrystallization of the orthorhombic $\mathrm{GUAPbI}_{3}$ phase on the substrates. The results of the morphological investigation on a typical $\mathrm{GUAPbI}_{3}$ layer are shown in Figure 6a-b. The tilted SEM image (Figure 6b) confirms the formation of a GUAPbI layer (d, in Figure 6b) on the $\mathrm{TiO}_{2}$ - FTO glass substrate (indicated with c, b and a respectively in Figure 6b) whereas the top view SEM image (Figure 6a) confirms the homogeneity of the layer with the presence of hollows possibly due to the evaporation of the solvent. The solar cell devices were fabricated according to the method reported by Eperon et al. [35]. The hole-transporting layer was then deposited by spin-coating a solution in chlorobenzene of 2,2',7,7'-tetrakis(N,N'-di-p-methoxyphenylamine)9,9'-spirobifluorene (spiro-OMeTAD), with additives of lithium bis-(trifluoromethanesulfonyl)imide (added in acetonitrile solution) and 4-tert-butylpyridine. Finally gold electrodes were thermally evaporated under vacuum. The current-voltage characteristics measured 

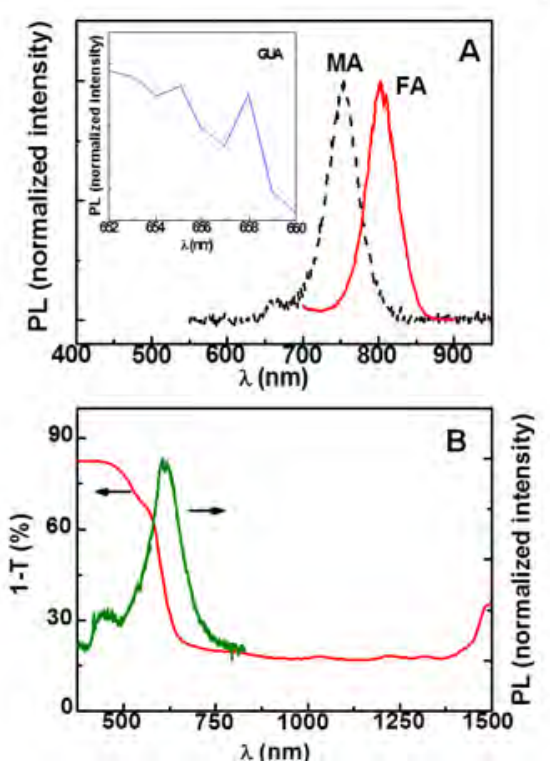

Figure 5. A) Normalized photoluminescence spectra of $\mathrm{FAPbI}_{3}$ and $\mathrm{GUAPbI}_{3}$ systems, after excitation with a Xe-arc lamp. The curve of $\mathrm{MAPbI}_{3}$ (black dots, Ref. [14]) has been added for comparison. B) Optical transmission and normalized photoluminescence spectra of $\mathrm{GUAPbI}_{3}$ system after excitation with a $\mathrm{Nd}$ $\operatorname{laser}(\lambda=355 \mathrm{~nm})$.
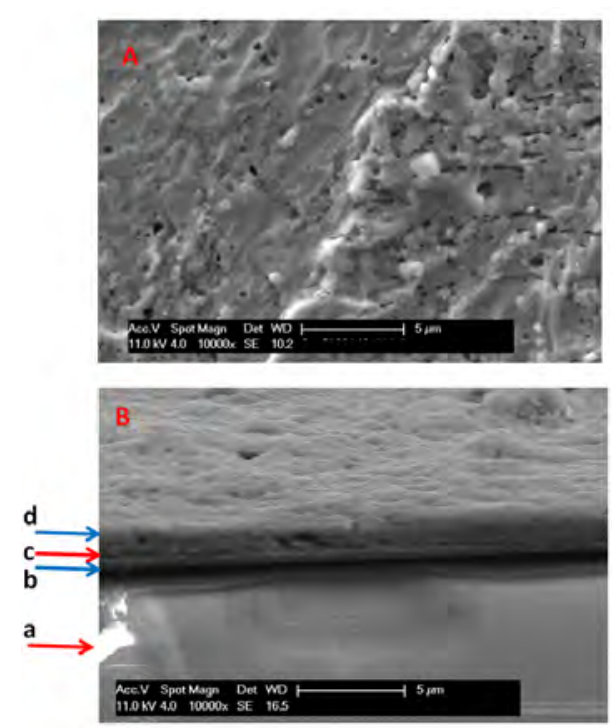

Figure 6. A) Top view and B) tilt view SEM images of a $\mathrm{GUAPbI}_{3}$ layer deposited on FTO glass substrate by doctor-blade, then by thermal annealing at $150^{\circ} \mathrm{C}$ for 5 minutes from $30 \%$ wt dispersion in DMSO. The thickness of the film is around $1.5 \mu \mathrm{m}$. The arrows indicate: a) glass substrate, b) ITO-layer; c) $\mathrm{TiO}_{2}$ layer; d) $\mathrm{GUAPbI}_{3}$ layer respectively.

under simulated sunlight (AM1.5, $100 \mathrm{~mW} \mathrm{~cm}^{-2}$ ) illumination from the best performing device with full device parameters extracted are shown in Figure 7. The J-V curves under illumination (black curves in Figure 7a) show average power conversion efficiency of $0.3 \%$ (inset table in Figure 7 ) which confirms the photoluminescence profile observed during our measurements. According to our data and the theoretical predictions of Giorgi et al. [21], its thermodynamic stability, low formation energy, and band gap make $\mathrm{GUAPbI}_{3}$ a potential candidate for future photovoltaic applications. The introduction of a third $\mathrm{NH}_{2}$ group results in very interesting and useful features in the final perovskite, which is expected to frustrate the more bulky guanidinium cation rotation in the semiconductor cavity.

\section{Conclusions}

In this work, formamidinium and guanidinium containing lead iodide perovskites have been investigated XRD 


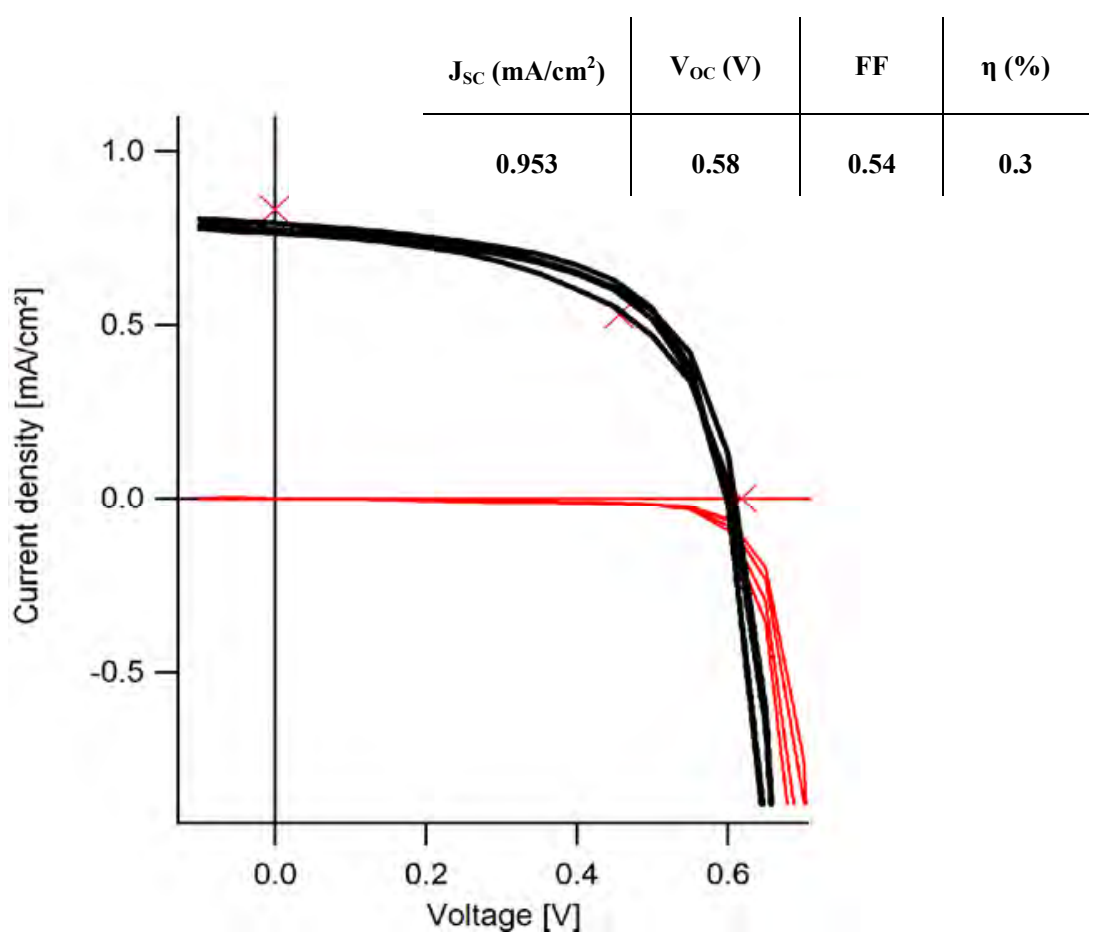

Figure 7. $\mathrm{J}-\mathrm{V}$ characteristics measured under simulated sunlight (AM1.5, $100 \mathrm{~mW} \mathrm{~cm}{ }^{-2}$ ) illumination from the best performing GUAPbI3 solar cell with full device parameters extracted (inset table). The black curves are obtained under illumination of the solar cell whereas the red curves are obtained without illumination of the device.

analysis reveals the formation, at room temperature, of a trigonal structure (black color) ( $\alpha-\mathrm{FAPbI}_{3}$, space group P3m1) and of a non-perovskite structure with hexagonal structure (yellow color) ( $\delta$-FAPbI ${ }_{3}$, space group $\left.\mathrm{P} 63 m c\right)$ for formamidinium containing perovskites, of an orthorhombic structure (space group Pnma) and of an hexagonal structure (as secondary phase) for $\mathrm{GUAPbI}_{3}$ which indicate a decrease of the symmetry of the crystalline structure by increasing the cation ionic radius.

The morphological investigation revealed the growth of aggregate crystallites for the $\alpha-\mathrm{FAPbI}_{3}$ phase due fast separation from the mother liqueur and to the high stirring. On the other hand, fiber-like crystallites for $\mathrm{GUAPbI}_{3}$ up to 10-12 $\mathrm{mm}$ have been observed because of the slow cooling and the discontinued stirring. The infrared spectra (IR) of the crystalline powders were measured at room temperature. The IR-spectra confirmed the influence of the additional C-N bonds in the organic cations and of the stronger electron delocalization was observed in FA and GUA containing systems. 
Optical absorption measurements indicate that, $\mathrm{FAPbI}_{3}$ and $\mathrm{GUAPbI}_{3}$ systems behave as direct-gap semiconductors with energy band gaps of $1.42 \mathrm{eV}$ and $2.16 \mathrm{eV}$ respectively at room temperature. Photoluminescence measurements showed the presence of band-to-band radiative recombination. The behavior of the guanidinium containing system could be explained by the preferential formation of low-dimensional structures as well as through enhanced hydrogen bonding to the inorganic matrix altering the covalent/ionic character of $\mathrm{Pb}-\mathrm{I}$ bonds and consequently increasing the shrinkage of the final cell.

The prepared powders were used as precursors to prepare layers deposited on FTO coated glass substrates by spin-coating and thermal treatment. The morphological investigation revealed the formation of a homogeneous yellow layer of $\mathrm{GUAPbI}_{3}$ with the presence of small hollows possibly due to the fast evaporation of the solvent during the thermal treatment. The current-voltage characteristics measured under simulated sunlight (AM1.5, 100 $\mathrm{mW} \mathrm{cm}{ }^{-2}$ ) illumination from the best performing device show average power conversion efficiency of $0.3 \%$.

\section{Acknowledgements}

Many thanks are owed to Mr. J.-C. Jaud for technical assistance during XRD analysis and Ms. K. Lakus-Wollny for the technical assistance in the SEM analysis.

The authors thank the Federal Ministry of Research and Development (BMBF) (Project "Perosol” Nr. 03SF0483B) for the financial support during this work.

\section{Notes}

$\dagger$ Electronic Supplementary Information (ESI) available: [Short report on the calculation of the $t$ and $\mu$ factors; Table S1: FT-IR group frequencies/assignments for $\mathrm{FAPbI}_{3}$ and $\mathrm{GUAPbI}_{3}$ systems obtained from the spectra shown in Figure 3a-b; Figure S1: XRD pattern and SEM image of $\alpha-\mathrm{FAPbI}_{3}$ phase; Figure S2: XRD pattern and IR-spectrum of $\delta-\mathrm{FAPbI}_{3}$ phase]. 


\section{References}

[1] M. Grätzel Nature Materials 13 (2014) 838-842.

[2] M.A. Green, A. Ho-Baillie, H.J. Snaith Nature Photonics 8 (2014) 506-514.

[3] C.R. Kagan, D.B. Mitzi, C. Dimitrakopoulos Science 286 (1999) 945-947.

[4] F. Bassani, G.C. La Rocca, V.M. Agranovich Int. J. Quantum Chem. 77 (2000) 973-981.

[5] G. Vijaya Prakash, K. Pradeesh, R. Ratnani, K. Saraswat, M.E. Light, J.J. Baumberg J. Phys. D: Appl. Phys. 42 (2009) 185405.

[6] K. Pradeesh, J.J. Baumberg, G. Viajaya Prakash Appl. Phys. Lett. 95 (2009) 033309.

[7] C.K. Moller, Nature 180 (1957) 981.

[8] C.K. Moller, Nature 182 (1958) 1436.

[9] D.Z. Weber, Naturforsch. 33b (1978) 862.

[10] D.Z. Weber, Naturforsch., 33b (1978) 1443.

[11] J.H. Im, J. Chung, S.J. Kim, N.G. Park Nanoscale Res. Lett. 7 (2012) 353.

[12] N.K. McKinnon, C.D. Reeves, M.H. Akabas J. Gen. Physiol. 138 (2011) 453-466.

[13] G.E. Eperon, S.D. Stranks, C. Menelaou, M.B. Johnston, L.M. Herz, H.J. Snaith Energy Environ. Sci. 7 (2014) 982-988.

[14] L. Dimesso, M. Dimamay, M. Hamburger, W. Jaegermann Chem. Mater. 26 (2014) 6762-6770.

[15] G. Kortüm, W. Braun, G Herzog Angew. Chem. 75 (1963) 653-661.

[16] T.J. McCarthy, T. A. Tanzer, M.G. Kanatzidis J. Am. Chem. Soc. 117 (1995) 1294-1301.

[17] V.M. Goldschmidt Die Naturwissenschaften 21 (1926) 477-485.

[18] C. Li, X. Lu, W. Ding, L. Feng, Y. Gao, Z. Guo Acta Cryst. B64 (2008) 702-707.

[19] A.M. Glazer Acta Crystallogr. Sect. B: Struct. Crystallogr. Cryst. Chem. 28 (1972) 3384-3392.

[20] G. Kieslich, S. Sun, A.K. Cheetham Chem. Sci. 5 (2014) 4712-4715.

[21] G. Giorgi, J.-I. Fujisawa, H. Segawa, K. Yamashita J. Phys. Chem. C 119 (2015) 4694-4701.

[22] C.C. Stoumpos, C. D. Malliakas, M.G. Kanatzidis Inorg. Chem. 52 (2013) 9019-9038.

[23] M.T. Weller, O.J. Weber, J.M. Frost, A. Walsh J. Phys. Chem. Lett. 6 (2015) 3209-3212. 
[24] C.L. Angell, N. Sheppard, A. Yamaguchi, T. Shimanouchi, T. Miyazawa, S. Mizushima Trans. Faraday Soc. 53 (1957) 589-600.

[25] M. Szafranski Thermochim. Acta 307 (1997) 177-183.

[26] M. Szafranski, A. Katrusiak Phys. Rev. B 61 (2000) 1026-1035.

[27] M. Grottel, M. Szafranski, Z.Z. Pajak Naturforsch. 52a (1997) 783-788.

[28] N. Preda, L. Mihut, M. Baibarac, I. Baltog, R. Ramer, J. Pandele, C. Andronescu, V. Fruth J. Mater. Sci.:

Mater. Electron. 20 (2009) S465-S470.

[29] M. Drozd Mater. Sci. Eng. B 136 (2007) 20-28.

[30] T. Todorova, O. Kröcher, B. Delley J. Molec. Struc.: THEOCHEM 907 (2009) 16-21.

[31] E. Kucharska, J. Hanuza, A. Ciupa, M. Maczka, L. Macalik Vibrational Spectroscopy 75 (2014) 45-50.

[32] N. Ghobadi Internat. Nano Lett. 3 (2013) 2.

[33] N. Pellet, P. Gao, G. Gregori, T.Y.Yang, M.K. Nazeeruddin, J. Maier, M. Grätzel, Angew. Chem. Int. Ed. 53 (2014) 3151-3157.

[34] P.E. Mason, G. W. Neilson, C. E. Dempsey, A. C.; Barnes, J.M. Cruickshank, Proc. Natl. Acad. Sci. USA 100 (2003) 4557.

[35] G. E. Eperon, D. Bryant, J. Troughton, S. D. Stranks, M. B. Johnston, T. Watson, D. A. Worsley, H. J. Snaith J. Phys. Chem. Lett. 6 (2015) 129-138. 
Graphical Abstact

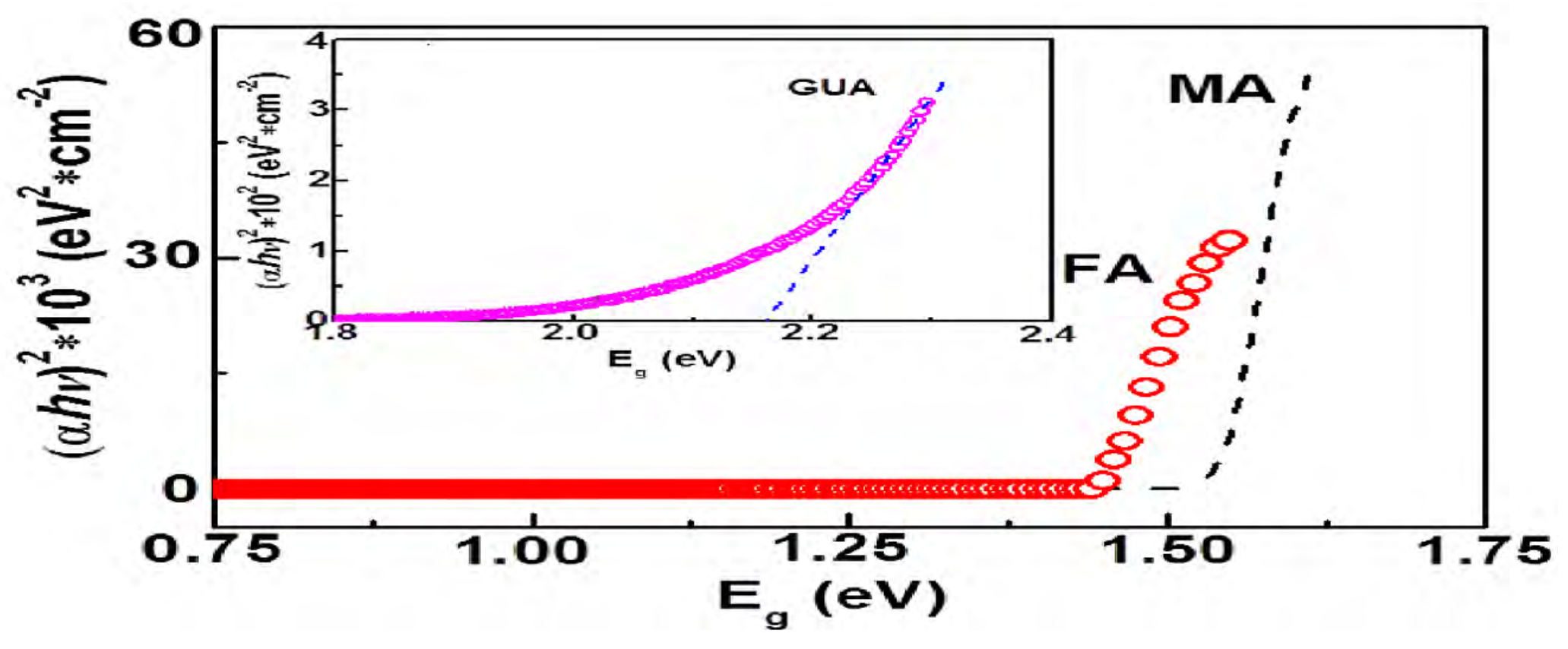

